\title{
La réponse en fréquence comme signature d'usinabilité
}

\section{Proposition de méthodologie}

\author{
Alain Giraudeau ${ }^{\mathrm{a}}$ et Mohamed El Mansori
}

Laboratoire de Mécanique et Procédés de Fabrication (EA4106), École Nationale Supérieure d'Arts et Métiers, Rue Saint-Dominique, BP 508, 51006 Châlons-en-Champagne, France

Reçu le 22 octobre 2006, accepté le 27 juin 2007

\begin{abstract}
Résumé - L'article propose une méthodologie basée sur une procédure expérimentale originale utilisant le comportement vibratoire du système de coupe pour dégager des indicateurs d'usinabilité de matériaux. Elle est appliquée à un outil de tournage en opération de chariotage. Le bridage de l'outil et les phénomènes liés à la coupe définissent les conditions aux limites de l'outil assimilé à une structure vibrante. Toute variation des conditions de coupe se répercute sur le comportement vibratoire de l'outil. Ce comportement peut être représenté par les fonctions de réponse en fréquence de l'outil déterminées pendant l'usinage. Ces dernières sont calculées à partir d'excitations percussionnelles et des réponses vibratoires de l'outil mesurées pendant les opérations de coupe. Un dispositif spécifique d'excitation est utilisé. Sa conception et sa validation sont présentées. Un dépouillement particulier issu des techniques d'analyse modale expérimentale est appliqué à des mesures exploratoires. Les résultats présentés montrent la sensibilité de la méthode. Des perspectives de développement sont avancées.
\end{abstract}

Mots clés : Usinabilité / coupe / fonction de réponse en fréquence / excitation percussionnelle / analyse fréquentielle

\begin{abstract}
The frequency response as machinability signature. Proposal for a methodology. The paper proposes a novel methodology based on an original experimental procedure which uses the vibrating behaviour of the cutting system to provide indicators on the machinability of materials. A lathe tool in straight turning operation is considered. Fastening and cutting define the boundary conditions of the tool considered as a vibrating structure. Any change under the cutting conditions is reflected on the vibrating behaviour of the tool. This behaviour can be represented by the tool frequency response functions measured in operation. These latter are computed from impact excitations and vibrating responses measured during the cutting operations. The design and the validation of the specific device used for the excitation are presented. A basic estimation of the modal parameters of the tool is extracted from the data. First results of preliminary tests in cutting metallic materials are presented. These results show an interesting sensitivity of the method. Several improvements are proposed and discussed.
\end{abstract}

Key words: Machinability / material cutting / frequency response function / impact excitation / vibrating analysis

\section{Introduction}

Les vibrations générées en usinage se traduisent généralement, sur la pièce, par un mauvais état de la surface usinée et, sur l'outil, par une destruction prématurée de l'arête de coupe. Elles sont dues essentiellement aux phénomènes physiques liés au processus dynamique de la coupe et à la cinématique de la machine-outil. On

a Auteur pour correspondance :

alain.giraudeau@chalons.ensam.fr constate en général que le comportement vibratoire du système de coupe, pour des conditions d'usinage données, sera d'autant plus influencé que le processus de coupe engendre $[1,2]$ :

- un frottement variable à l'interface outil-copeau qui est fonction de la vitesse du copeau, et lui-même fonction de plusieurs paramètres opératoires;

- un contact fragile ou talonné entre la face de dépouille de l'outil et de la pièce et dont l'angle de dépouille a une influence directe sur l'amplitude des vibrations; 


\section{Nomenclature}

\begin{tabular}{|ll|}
\hline$F(f):$ & Spectre discret de l'effort d'excitation percussionnelle. Fonction complexe. \\
$H_{k l}(f):$ & Fonction de réponse en fréquence de la structure exprimée entre les degrés de liberté $k$ et $l$. \\
& Fonction complexe. \\
$H_{1}(f):$ & Estimateur permettant le calcul de la fonction de réponse en fréquence (F.R.F.) à partir \\
& de mesures effort et réponse vibratoires. Fonction complexe. \\
$S_{\mathrm{FF}}(f):$ & Autospectre de l'effort d'excitation percussionnelle. Fonction réelle. \\
$S_{\mathrm{XF}}(f):$ & Interspectre de l'effort d'excitation percussionnelle et de la réponse vibratoire à l'excitation seule. \\
& Fonction complexe. \\
$S_{\mathrm{XX}}(f):$ & Autospectre de la réponse vibratoire à l'effort d'excitation seule. Fonction réelle. \\
$X_{\mathrm{usin}}(f):$ & Spectre discret de la réponse vibratoire à l'usinage seul. Fonction complexe. \\
$X_{\mathrm{choc}}(f):$ & Spectre discret de la réponse vibratoire à l'effort d'excitation percussionnelle superposé \\
& à l'effort usinage. Fonction complexe. \\
$\gamma^{2}(f):$ & Fonction de cohérence, adimensionnelle variant entre 0 et 1 et fournissant un indicateur \\
& du rapport signal/bruit de l'estimation de la F.R.F. Fonction réelle. \\
\hline
\end{tabular}

- une pente aléatoire de la surface à usiner par rapport à la vitesse de coupe. Celle-ci modifie l'angle de cisaillement et par suite le processus de formation du copeau. La variation de cet angle a un effet direct sur les oscillations de la bande de cisaillement;

- un mouvement oscillatoire de l'outil qui entraîne une variation de l'angle de coupe ainsi que celui de dépouille;

- une épaisseur variable de la coupe liée à des ondulations de la surface libre à usiner. Ceci conduit à des variations de la quantité de matière enlevée impliquant ainsi des efforts de coupe fluctuants qui auto-excitent le système.

L'ensemble de ces effets est fortement couplé et nonlinéaire. Leur discernement par des modèles simplifiés est forcément éloigné de la réalité physique du problème. Les modèles dynamiques développés en coupe, pour leur prise en compte, restent en effet très parcellaires. Ils sont basés essentiellement soit sur $[2,3]$ :

- une approche linéaire par les fonctions de transfert qui prennent en compte l'ondulation de la surface à usiner et les mouvements de l'outil ;

- une approche analytique qui prend en compte les variations du coefficient de frottement moyen à l'interface outil-copeau en fonction de la vitesse relative du copeau par rapport à l'outil ;

- une approche non-linéaire qui permet de préciser partiellement le comportement vibratoire du système de coupe en prenant en compte certaines non-linéarités dues par exemple à la variation de sortie de l'outil associée à l'interférence entre la face en dépouille de l'outil et les ondulations de la surface usinée.

S'y ajoute aussi une difficulté supplémentaire liée à l'exploitation même de ces modèles [3-6]. Celle-ci repose principalement sur l'identification expérimentale qui revient à déterminer d'une manière générale trois types de paramètres : les paramètres dynamiques correspondant au mouvement de l'outil, équivalence en terme de masse, raideur et amortissement, les paramètres pilotant la loi de frottement à l'interface outil-copeau et enfin les paramètres dépendant du matériau usiné. Les paramètres dynamiques sont déterminés classiquement par essais statiques pour la rigidité équivalente et par la réponse dynamique du système outil-porte-outil à une impulsion donnée à l'aide d'un marteau d'impact. Compte tenu des hypothèses introduites dans l'approche des fonctions de transfert, l'identification ne peut être très satisfaisante. L'identification de la loi de frottement est issue généralement des essais de coupe instrumentée en efforts par capteur piézoélectrique. Elle admet par ailleurs une hypothèse approximative assignant que le frottement moyen ne dépend que de la vitesse de défilement du copeau. Enfin, se pose le problème de l'identification de la loi de comportement du matériau usiné à grande vitesse de déformation et plus particulièrement de la contrainte de cisaillement en coupe.

De ces éléments relatifs à la connaissance du comportement vibratoire de l'ensemble outil-matière, retenons ici :

- que les phénomènes vibratoires en usinage sont souvent abordés sous l'aspect de leur prédiction, de leur contrôle ou de leurs effets.

- que les modèles développés ne prennent pas vraiment en compte les problèmes de couplage liés aux phénomènes des vibrations en usinage. De plus le développement d'études paramétriques sur ces modèles se heurtent au choix numérique des paramètres, à l'analyse de sensibilité, et autres ;

- que l'analyse vibratoire en usinage d'un point de vue expérimental est souvent réduite à la portion congrue. La réponse vibratoire se limite généralement à celle du système outil-porte outil obtenue de manière préopératoire et qui ne prend pas en compte les conditions aux limites (conditions de coupe);

- qu'il est malaisé d'appréhender les phénomènes vibratoires inhérents à la coupe si l'on n'a pas décrit très complètement et sous une procédure reproductible : - en quoi consiste la structure du système de coupe; 
- comment le comportement vibratoire intrinsèque de cette structure peut être décrit par la fonction de réponse en fréquence.

Telles sont les raisons qui ont motivé cette étude.

\section{Méthode proposée}

\subsection{Principe}

La méthode cherche à utiliser le comportement vibratoire soit de l'outil soit celui de la pièce comme vecteur d'information. Elle consiste en une adaptation, spécifique à la problématique des techniques classiques d'analyse vibratoire, tant sur le plan de la mesure que de leur exploitation.

Le comportement vibratoire d'un outil de tournage en opération de chariotage est ici considéré. Très généralement le comportement d'une structure est déterminé par la géométrie de celle-ci, par les caractéristiques des matériaux la constituant, la nature des liaisons internes et par les conditions aux limites imposées.

Dans le cas de l'outil considéré, les conditions aux limites sont d'une part celles qui correspondent à la fixation de l'outil dans son porte-outil, et d'autre part constituées, en pointe d'outil, par l'ensemble des phénomènes liés à la coupe.

La procédure expérimentale assure l'invariabilité de toutes les autres conditions. En particulier la pièce usinée est suffisamment massive pour que l'enlèvement de matière ne modifie pas ses caractéristiques de manière appréciable. Une modification du comportement vibratoire de l'outil pendant les opérations d'usinage ne peut être alors que la conséquence d'un changement des conditions de coupe. Une quantification du comportement vibratoire intrinsèque de l'outil peut alors fournir un indicateur sur les conditions de l'usinage.

\subsection{Fonction de réponse en fréquence (F.R.F.)}

Cette fonction $H_{k l}(f)$ est classiquement définie entre n'importe quel couple de degrés de liberté, $k$ et $l$, de la structure telle que :

$$
H_{k l}(f)=\frac{F_{k}(f)}{X_{l}(f)}=\frac{F_{l}(f)}{X_{k}(f)}
$$

Dans laquelle $X_{k}(f)$ est la réponse, dans le domaine des fréquences, du degré de liberté $k$ lorsque l'effort d'excitation $F_{l}(f)$, exprimé également dans le domaine des fréquences, s'exerce sur le degré de liberté $l$. Réciproquement, $X_{l}(f)$ est la réponse du degré de liberté $l$, lorsque la structure est excitée suivant le degré de liberté $k$ par l'effort $F_{k}(f)$. Cette fonction est intrinsèque à la structure ainsi qu'aux conditions aux limites qui lui sont imposées. La méthode repose sur la capacité à déterminer expérimentalement les F.R.F. de l'outil pendant les opérations d'usinage, à partir des vibrations de l'outil mesurées respectivement dans les directions de coupe et d'avance, en réponse à des excitations contrôlées et exercées dans les mêmes directions.

Les représentations numériques, dans le domaine des fréquences de l'excitation et des réponses vibratoires, sont calculées par la Transformée de Fourier Discrète (algorithme «FFT») appliquée à des blocs d'échantillons numériques acquis à partir des signaux correspondants. Comme dans toutes analyses fréquentielles par moyens numériques, les opérations de pondérations temporelles, moyennages et autres, doivent être appliquées. La spécificité de la présente application ne permet pas l'utilisation directe des fonctions généralement présentes dans les analyseurs du commerce. Un traitement particulier des acquisitions a été développé sous Matlab $^{\text {TM }}$.

\section{Analyse de l'expérimentation}

\subsection{Mesure des réponses vibratoires}

Les vibrations de l'outil sont mesurées à l'aide de deux accéléromètres miniatures, fixés dans le corps de l'outil aussi près que possible de la plaquette de coupe, à l'aide de deux goujons miniatures. Ils mesurent les accélérations absolues dans les directions de coupe et d'avance.

\subsection{Choix du type d'excitation}

Il est, a priori, préférable de ne pas réaliser l'estimation des fonctions de réponse en fréquence de l'outil à partir de la seule excitation provenant de l'effort de coupe :

- les composantes de celui-ci sont éventuellement mesurables au niveau du porte-outil mais leurs contenus fréquentiels ne peuvent être maîtrisés. Le rapport signal/bruit peut se révéler faible dans certaines bandes de fréquences et l'estimation des F.R.F. en sera dégradée;

- les indicateurs de coupe obtenus à partir de ces mêmes efforts manqueraient d'objectivité.

L'adaptation de l'analyse percussionnelle, classiquement utilisée en analyse vibratoire des structures a été préférée. Il faut noter que l'effort provenant de l'impact exercé sur l'outil pendant l'usinage, se superpose à l'effort de coupe. Il conviendra d'en séparer les effets lors de l'exploitation des mesures des réponses vibratoires de l'outil.

\subsection{Excitation percussionnelle}

Celle-ci est généralement réalisée manuellement à l'aide d'un marteau instrumenté. L'effort d'impact est mesuré par un capteur d'effort à grande bande passante, monté entre le corps du marteau et l'embout d'impact. La figure 1 montre un exemple de signal fourni par le 

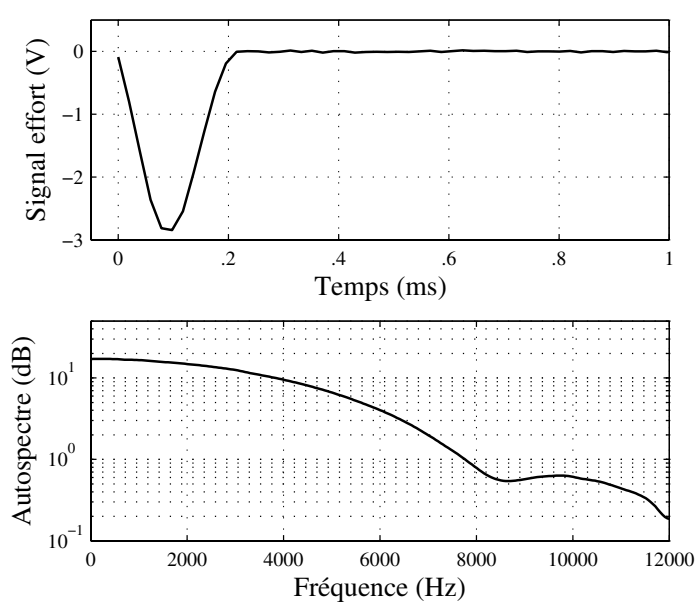

Fig. 1. Choc embout dur sur structure massive.
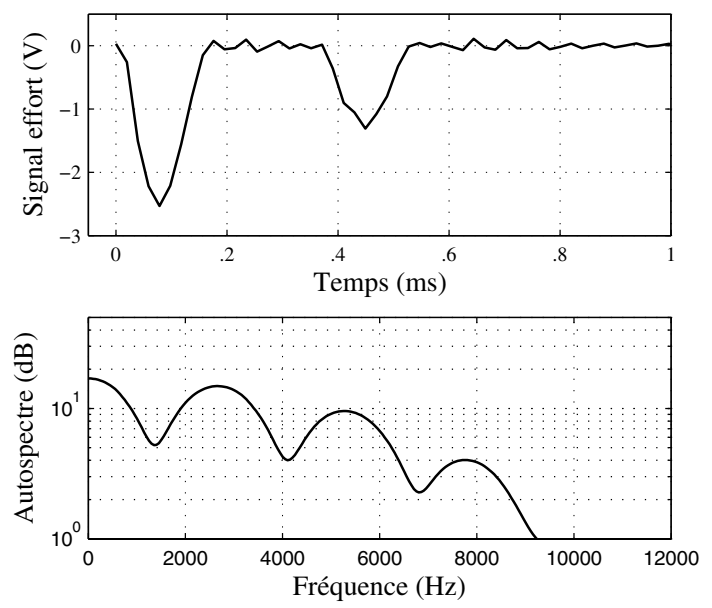

Fig. 2. Choc embout dur sur outil monté.

capteur d'un tel marteau équipé d'un embout dur en acier (56 HRC) suite à un choc manuel sur une structure massive.

Les représentations temporelles et fréquentielles de ce signal montrent un choc unique, sans rebond. La dureté de l'embout permet un choc élastique avec une très faible déformation de celui-ci, ce qui limite la durée du contact. En conséquence, le choc fournit à la structure une énergie vibratoire continûment répartie entre 0 et $6 \mathrm{kHz}$.

La figure 2 montre les représentations temporelles et fréquentielles du signal issu du même marteau, obtenu cette fois-ci suite à un impact manuel hors usinage sur un outil couteau monté dans le porte-outil d'un tour conventionnel.

On constate un choc double, suite à un rebond, dû à la relative souplesse de l'outil. Ceci entraîne un « allongement apparent $»$ du choc et par conséquent un spectre d'excitation perturbé. La réalisation manuelle d'impacts pendant les opérations d'usinage, conduirait à des excitations peu fiables et peu répétables. Enfin, la détermination des F.R.F. s'opère sur des moyennes de plusieurs acquisitions des signaux effort d'excitation et réponses de la structure. Ces acquisitions multiples sont à réaliser ici dans le temps d'une passe d'usinage. Ces différentes considérations ont entraîné la conception et la réalisation d'un dispositif spécifique d'excitation percussionnelle.

\section{Dispositif d'excitation percussionnelle}

\subsection{Analyse fonctionnelle}

Les différentes fonctions à remplir par le dispositif sont de :

- générer des impacts simples, sans rebonds, aussi brefs que le permettent la nature de l'embout et celle de la surface impactée;

- permettre des impacts répétitifs tant en localisation qu'en niveau d'effort et en direction d'impact, cette dernière devant être normale à la surface cible;

- permettre plusieurs impacts (8 à 10 minimum) dans la durée d'une passe d'usinage;

- pouvoir se fixer sur la tourelle porte-outil de manière à pouvoir impacter l'outil au choix dans les directions de coupe et d'avance.

\subsection{Principe et réalisation}

Le principe retenu est celui d'un système masseressort-amortisseur ne possédant qu'une seule mobilité en translation, en oscillations libres suite à un laché après déformation initiale du ressort. Pour obtenir un impact unique, seule la première élongation maximale doit permettre le contact embout-outil. Ceci est obtenu par les réglages de :

- la position d'équilibre de l'impacteur par rapport à l'outil ;

- l'extension initiale du ressort de rappel à l'instant du laché;

- l'amortissement introduit dans la liaison glissière.

Les risques de rebond dus aux vibrations de l'outil sont limités par le rappel énergique du ressort. Le mobile impacteur est une tige guidée en translation par deux glissières à billes. L'embout d'impact en acier est rigidement lié à une extrémité de la tige par l'intermédiaire du capteur d'effort. L'autre extrémité porte un embout métallique permettant une liaison magnétique temporaire avec un vérin pneumatique double effet dont l'axe est parallèle à celui de la glissière. Une butée réglable définit la compression des ressorts à l'instant du laché. Le schéma de principe du dispositif est présenté en figure 3 .

Pour réaliser un impact, la chambre avant du vérin est mise sous pression, faisant reculer la tige jusqu'au contact de la butée. Le vérin continuant sa course, la liaison magnétique est alors rompue, libérant la tige qui impacte la cible sous l'effet des ressorts de rappel. La tige de vérin est déplacée par la mise sous pression de la chambre arrière de celui-ci. La liaison magnétique avec la tige de l'impacteur est alors rétablie. Le retour de cette dernière à sa position d'équilibre s'effectue par la mise 


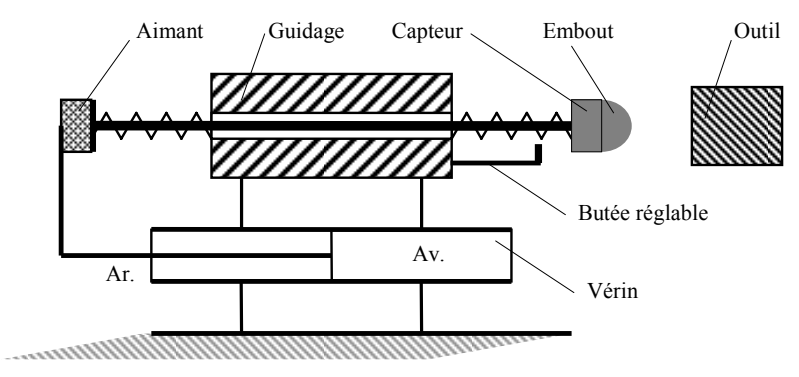

Fig. 3. Schéma du dispositif d'excitation.

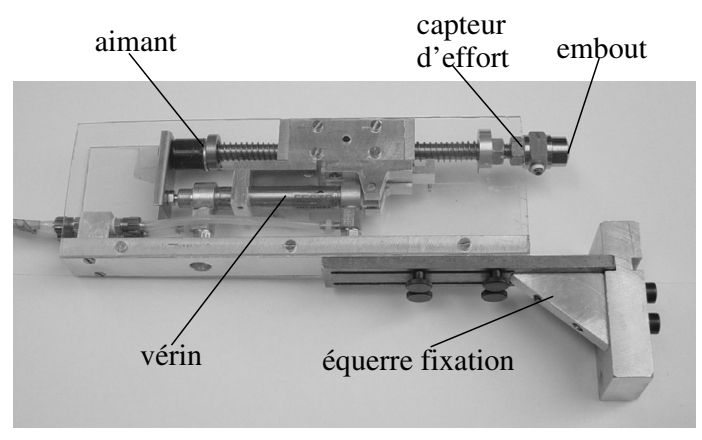

Fig. 4. Vue du dispositif d'excitation.

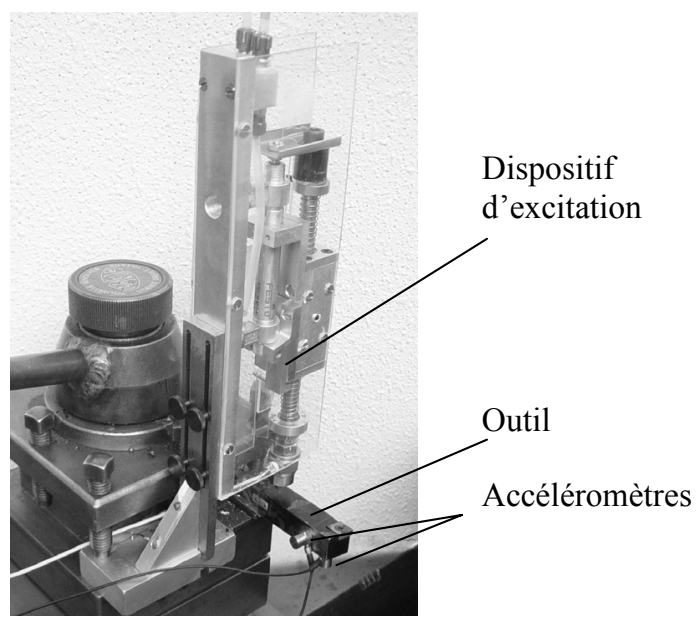

Fig. 5. Vue du dispositif d'excitation intégré au système de coupe.

hors pression des deux chambres du vérin. Un dispositif réglable de friction permet l'introduction d'une dissipation supplémentaire dans la liaison glissière de manière à obtenir l'unicité du choc.

Une vue du dispositif est présentée en figure 4.

La figure 5 montre une vue du dispositif monté sur la tourelle porte-outil, de manière à permettre la réalisation d'impacts sur l'outil dans la direction de coupe.

\subsection{Validation}

La figure 6 montre les représentations temporelles et fréquentielles du signal fourni par un impact sur l'outil couteau, monté dans le porte-outil du tour et réalisé à l'aide du dispositif décrit précédemment.
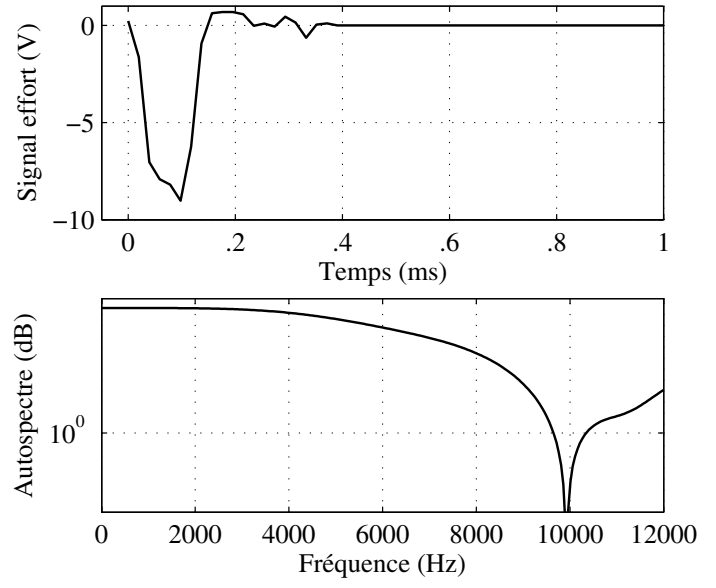

Fig. 6. Validation impacteur.

Sur la représentation temporelle, on observe un impact unique de durée légèrement inférieure à celui présenté figure 1. Des oscillations de faibles niveaux sont visibles à la suite du choc proprement dit. Une observation plus précise montre que celles-ci proviennent selon toute vraisemblance de la réponse du premier mode de vibrations longitudinales du solide impacteur. Leur fréquence est voisine de $8,5 \mathrm{kHz}$ ce qui correspond à la fréquence calculée du premier mode longitudinal d'une poutre librelibre possédant les mêmes caractéristiques. Dans ce cas ces oscillations enregistrées ne correspondent pas à des composantes d'effort appliqué à la cible. Elles sont donc éliminées par une pondération adaptée.

Avant application de la Transformée de Fourier Discrète (DFT) (algorithme «FFT») aux blocs d'échantillons acquis, une pondération leur est appliquée de manière à limiter les effets de la troncature temporelle des signaux $[7,8]$.

Les signaux efforts d'impact sont généralement pondérés par une fenêtre rectangulaire d'une durée voisine de $20 \%$ de la durée du bloc. Pour atténuer les oscillations mentionnées plus haut, une fenêtre rectangulaire particulière est employée. Elle est constituée du même nombre d'échantillons que les blocs acquis. Ceux-ci sont pris égaux à 1 pour les premiers termes correspondant à une durée voisine de $0,4 \mathrm{~ms}$ (durée majorée de l'impact) et nulle pour tous les autres termes. La pondération s'applique évidemment par simple multiplication terme à terme de la fenêtre et de chaque bloc acquis du signal effort impact.

La représentation fréquentielle de la figure 6 est celle du signal de l'effort d'impact ainsi traité. Elle est à comparer avec son homologue de la figure 1 . Ceci valide la qualité de l'excitation réalisée par le dispositif. L'expérience montre qu'il est aisé de réaliser plus d'un impact par seconde. Dans ces conditions la périodicité d'impact est surtout fonction de l'amortissement des réponses vibratoires de l'outil. Il est ainsi aisé d'effectuer des acquisitions multiples durant une passe d'usinage en vue de réalisations de moyennes. 


\section{Acquisitions}

De manière à garder la maîtrise la plus complète des traitements appliqués sur les signaux, seules les fonctions de filtrage anti-repliement et d'échantillonnage sont assurées par la chaîne d'acquisition. Lors des séquences d'une douzaine de secondes les signaux sont acquis en continu et les échantillons stockés en mémoire vive de la chaîne. Environ dix impacts commandés à distance par bouton poussoir sont généralement réalisés pendant cette durée. Les échantillons sont ensuite transférés dans la mémoire du calculateur. Une procédure spécifique et interactive permet alors de sélectionner et d'archiver des blocs de $2^{n}$ échantillons (typiquement 1024 ou 2048 échantillons) correspondant aux informations relatives aux impacts et à leurs réponses.

Des acquisitions de vibrations de l'outil sans impact et dans les mêmes conditions d'usinage sont également effectuées avec la même procédure.

\section{Détermination des fonctions de réponse en fréquence (F.R.F.)}

\subsection{Estimation des réponses aux impacts}

Pour tenir compte de la décroissance progressive des réponses vibratoires due aux différents phénomènes dissipatifs, une pondération exponentielle est généralement adoptée sur les réponses percussionnelles [7]. Une telle fenêtre a été utilisée dans cette étude. Le premier terme vaut 1 et le dernier 0,01 , la décroissance est exponentielle entre les deux extrêmes. Cette pondération a été appliquée de la même façon que précédemment aux blocs d'échantillons des signaux réponses vibratoires à l'impact. La même pondération exponentielle a été appliquée aux blocs de signaux acquis lors d'usinage sans impact.

Par application de la Transformée de Fourier Discrète aux blocs d'échantillons effort, réponses à l'impact et vibrations en usinage seul, on obtient respectivement $F(f)$, $X_{\text {choc+usin }}(f), X_{\text {usin }}(f)$ qui sont les vecteurs numériques contenant les spectres instantanés unilatéraux de ces trois signaux.

Comme préalablement indiqué, les réponses vibratoires de l'outil à l'impact se superposent aux vibrations de celui-ci dues à l'usinage. En faisant l'hypothèse d'un comportement vibratoire linéaire de l'outil, la réponse $X(f)$ de celui-ci au seul impact est estimée comme indiquée ci-après :

$$
X(f)=X_{\text {choc }+ \text { usin }}(f)-\frac{1}{n} \sum_{i=1}^{n}\left(X_{\mathrm{usin}}(f)\right)_{i}
$$

$n$ étant le nombre de blocs sélectionnés dans l'acquisition.

De manière à minimiser l'influence du bruit de mesure provenant des signaux des réponses vibratoires, l'estimation des F.R.F. a été effectuée à l'aide de l'estimateur $H_{1}(f)[7]$ tel que :

$$
H_{1}(f)=\frac{S_{\mathrm{XF}}(f)}{S_{\mathrm{FF}}(f)}
$$

Le rapport signal/bruit des F.R.F. est estimé à travers la fonction de cohérence $\gamma^{2}(f)$ telle que :

$$
\gamma^{2}(f)=\frac{\left|S_{\mathrm{XF}}\right|^{2}}{S_{\mathrm{FF}} \cdot S_{\mathrm{XX}}}
$$

\section{Essais exploratoires}

\subsection{Conditions expérimentales}

Des essais exploratoires ont été conduits avec le dispositif décrit précédemment et représenté en figure 5 . Ils ont été effectués avec un outil couteau chariotant un cylindre d'acier E335, de $65 \mathrm{~mm}$ de diamètre avec une vitesse de coupe de $130 \mathrm{~m} \cdot \mathrm{min}^{-1}$, une avance de $0,4 \mathrm{~mm} \cdot \mathrm{tr}^{-1}$ et une profondeur de passe de $1 \mathrm{~mm}$.

Les F.R.F. et fonctions associées ont été calculées à partir de blocs de 1024 échantillons provenant des signaux excitation et réponse vibratoire issus de huit acquisitions successives. Compte tenu de la bande d'analyse utilisée $(10 \mathrm{kHz})$, la résolution fréquentielle est limitée à $25 \mathrm{~Hz}$.

Afin de préciser la sensibilité de la méthodologie aux conditions de contact, les essais ont été conduits dans les mêmes conditions d'usinage en utilisant une plaquette neuve et une plaquette usée. Les réponses vibratoires ont été mesurées uniquement dans la direction de coupe suite à des impacts réalisés dans la même direction.

\subsection{Premiers résultats}

La fenêtre supérieure de la figure 7 montre l'autospectre de l'effort d'excitation, les fenêtres suivantes montrent les diagrammes de Bode des F.R.F. (modules et phases) et les fonctions de cohérence obtenus à partir des essais avec plaquette neuve (tracés continus) et plaquette usée (traits discontinus). Pour l'autospectre de l'excitation et le module de la F.R.F. les unités des ordonnées sont arbitraires mais identiques pour les deux essais.

Les courbes des diagrammes de Bode des F.R.F. issues des deux essais ne présentent pas de différences appréciables. Les courbes des modules présentent toutes les deux un pic prononcé dans la bande de fréquence centrée sur $2,4 \mathrm{kHz}$. À la même fréquence correspond une variation accentuée des deux courbes de phase des F.R.F. indiquant que cette bande de fréquence correspond à celle d'un mode vibratoire de l'outil. Des essais complémentaires ont confirmé qu'il s'agit du premier mode de flexion de celui-ci dans le plan vertical. La figure 8 présente un agrandissement des diagrammes de Bode dans la bande de fréquence [2,3-2,5 kHz], avec une échelle linéaire pour les ordonnées. Cette représentation 

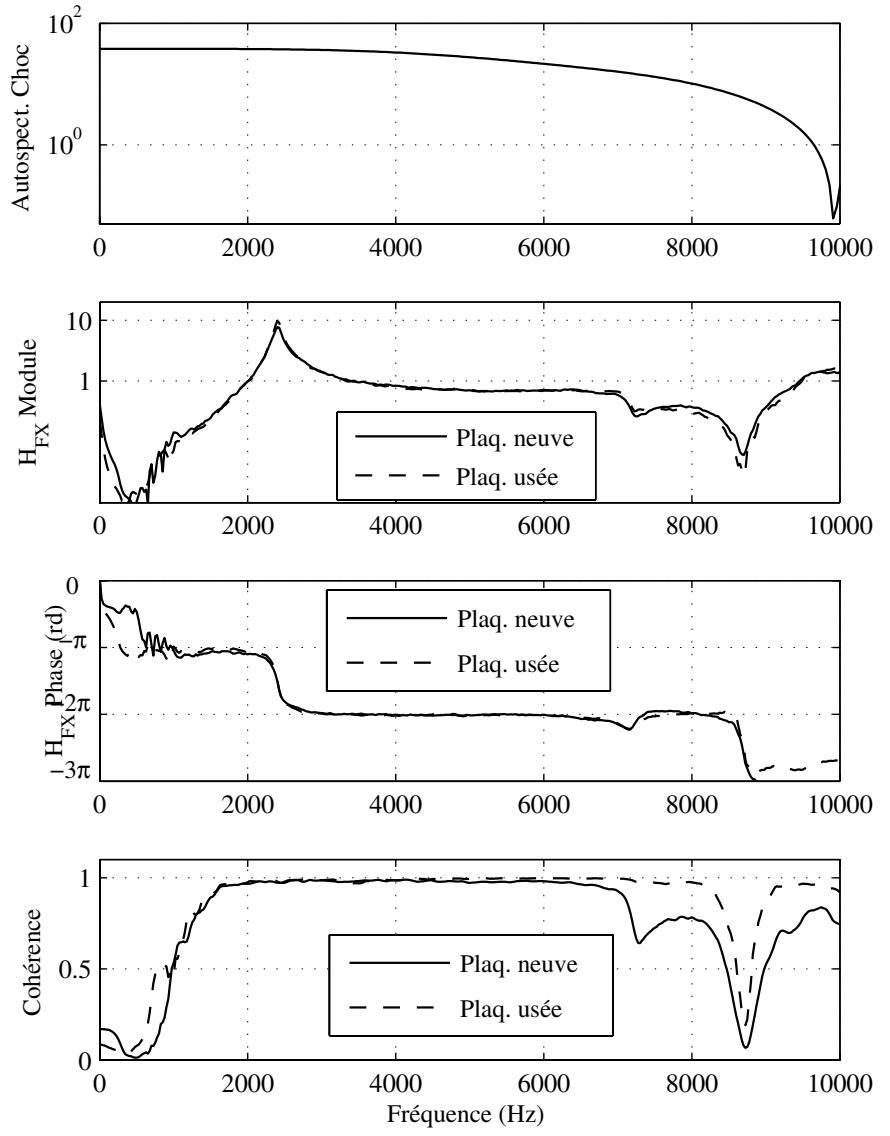

Fig. 7. Mesures exploratoires, Diagrammes de Bode et cohérence.

permet de faire apparaître une modification du comportement vibratoire de l'outil. Des essais complémentaires ont permis de montrer qu'elle se révèle répétitive. Toutefois, la résolution fréquentielle ne permet pas, dans le présent cas, de quantifier la variation de la fréquence propre entre les deux essais. La représentation de Bode ne permet pas non plus d'évaluer, de manière fiable, une variation appréciable de l'amplitude de la réponse, donc une modification de l'amortissement externe apporté à la structure par l'opération de coupe.

\section{Commentaires}

Les deux cohérences sont pratiquement égales à 1 dans la bande de fréquence $[2-6 \mathrm{kHz}]$. Ceci indique que les F.R.F. y sont obtenues avec un bon rapport signal/bruit. Les faibles réponses vibratoires de l'outil dans les basses fréquences ainsi qu'autour de $9 \mathrm{kHz}$ (Fig. 7) entraînent évidemment des rapports signaux/bruit faibles aux niveaux de leurs mesures et expliquent les effondrements constatés des deux cohérences dans ces bandes de fréquences.

Les remarques citées en section 7.2 montrent que la représentation de Bode, n'est certainement pas celle qui fournira le plus d'informations. La représentation de Nyquist des F.R.F. dans le plan complexe permet de

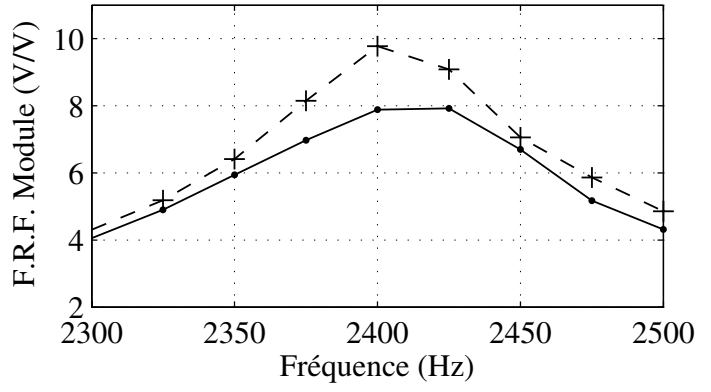

Plaq. neuve -
Plaq. usée $\quad----$

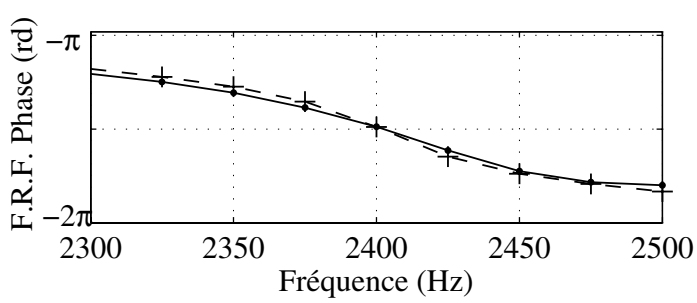

Fig. 8. Diagramme de Bode, agrandissement local.

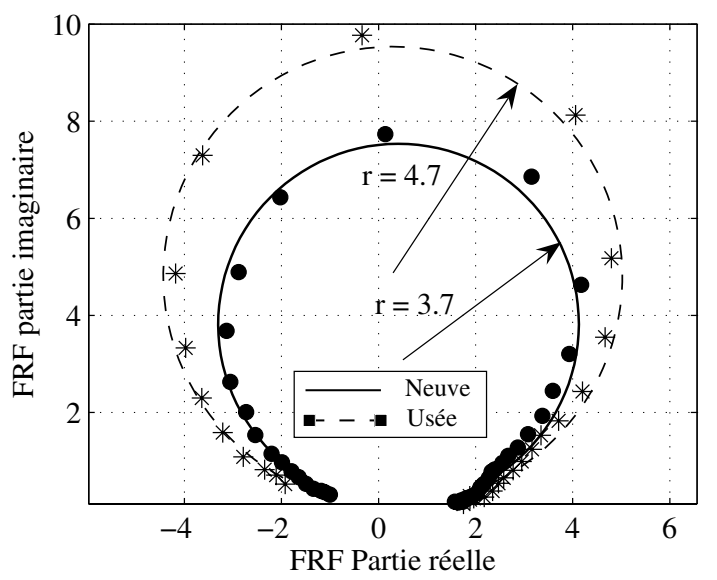

Fig. 9. Mesures exploratoires, diagrammes de Nyquist.

dilater considérablement l'information dans les bandes fréquentielles contenant un mode. D'un point de vue vibratoire, l'outil est une structure simple possédant une faible densité modale. Ceci est confirmé par le diagramme de Bode de la figure 7 qui ne montre l'existence que du seul mode de flexion précédemment cité dans la bande de fréquence $[0-5 \mathrm{kHz}]$. Il est alors possible d'appliquer une technique de dépouillement dite « SDOF » («Single Degree of Freedom») issue de l'analyse modale expérimentale $[9,10]$. Celle-ci permet de lisser par un cercle, dans le plan complexe, les valeurs discrètes des F.R.F. calculées dans une bande de fréquence entourant un mode. La valeur de la fréquence correspondant à l'intersection du cercle avec l'axe des imaginaires fournit une évaluation de la fréquence du mode [10]. Par ailleurs, il est montré que le rayon du cercle est directement lié à l'inverse de l'amortissement du mode.

La figure 9 montre les lissages par des cercles aux moindres carrés des deux F.R.F. précédentes dans une bande de fréquence de $400 \mathrm{~Hz}$ environ contenant le 
premier mode de flexion de l'outil dans le plan vertical. L'évaluation du glissement de la fréquence du mode reste délicate. Par contre la mesure des rayons des deux cercles, ici dans dans une unité arbitraire, permet une quantification de l'évolution de l'amortissement du mode entre les deux conditions d'usinage. Cette constatation montre la sensibilité de la méthode aux conditions d'usinage et permet d'entrevoir son utilisation comme un indicateur de l'usinabilité d'un matériau dans des conditions données.

\section{Conclusions}

La présente étude est une proposition de méthodologie d'utilisation des réponses vibratoires d'un outil comme vecteur d'information de l'usinabilité. Elle devra être comparée avec d'autres méthodes telles que le couple outil-matière (COM) ou le couple arête-matière (CAM) qui exploitent des mesures d'efforts de coupe et de puissance spécifique, ou encore le suivi d'usure de l'outil.

Des perspectives importantes d'améliorations de la méthode sont envisageables tant sur le plan de la mesure qu'au niveau du dépouillement :

- utilisation d'excitation forcée de type aléatoire ou multi-sinus pour accroître la résolution fréquentielle dans des bandes de fréquences dignes d'intérêt;

- mise en œuvre d'acquisitions simultanées avec différentes fréquences d'échantillonnage et tailles de blocs d'échantillons ;

- conception d'un corps d'outil spécifique permettant d'améliorer la sensibilité;

- mise en évidence des non-linéarités;

$-\ldots$

Un travail important demeure à accomplir avant la définition d'indicateurs d'usinabilité.

Des essais préliminaires d'usinage de matériaux différents permettent d'entrevoir une grande sensibilité de la méthode.
Remerciements. Les auteurs souhaitent remercier P.-Y. Bernard, Y. Legrand, A. Queant, R. Oury, élèves de l'I.T.I.I.C.A. pour leur large contribution à la conception et à la réalisation du dispositif impacteur.

\section{Références}

[1] D.A. Stephenson, J.S. Agapiou, Metal cutting theory and practice, Marcel Dekker Inc., New York, 1996

[2] R.I. King, Handbook of high-speed machining technology, Chapman and Hall Ltd, New York, 1985

[3] Y. Altintas, Manufacturing automation: metal cutting mechanics, Machine Tool vibrations, and CNC Design, Cambridge University Press, Paris, 2000

[4] W. Gong, W. Li, T. Shirakashi, T. Obikawa, An active method of monitoring tool wear states by impact diagnostic excitation, Int. J. Mach. Tools Manufact 44 (2004) 847-854

[5] J. Dong Yong, C. Young Gu, K. Hong-Gil, A. Hsiao, Study of the correlation between surface roughness and cutting vibrations to develop an on-line roughness measuring technique in hard turning, Int. J. Mach. Tools Manufact. 36 (1996) 453-464

[6] O.B. Abouelatta, J. Màdl, Surface roughness prediction based on cutting parameters and tool vibrations in turning operations, J. Materials Processing Technology 118 (2001) 269-277

[7] H. Herlufsen, Dual Channel FFT Analysis (Part 1), Bruël-Kjaer Technical review, Naerum, 1984

[8] P.M. Beaufils, M. Rami, Le filtrage numérique, Sybex, Paris, 1988

[9] R.J. Allemang, D.L. Brown, Handbook on Experimental Mechanics, Kobayashi, Seattle, 2nd edition, 1993

[10] D.J. Ewins, Modal Testing: Theory, Practice and Application, John Wiley and Sons, New York, 2nd edition, 2000 